\title{
Synthesis of Green Copper Nanoparticles Using Medicinal Plant Hagenia abyssinica (Brace) JF. Gmel. Leaf Extract: Antimicrobial Properties
}

\author{
H. C. Ananda Murthy $\mathbb{D}^{1},{ }^{1}$ Tegene Desalegn, ${ }^{1}$ Mebratu Kassa, ${ }^{1}$ Buzuayehu Abebe $\mathbb{D}^{\mathbb{D}}{ }^{1}$ \\ and Temesgen Assefa ${ }^{2}{ }^{2}$ \\ ${ }^{1}$ Department of Applied Chemistry, School of Applied Natural Science, Adama Science and Technology University, P.O. Box, \\ 1888 Adama, Ethiopia \\ ${ }^{2}$ Department of Biotechnology, College of Natural and Computational Science, Debre Berhan University, Ethiopia \\ Correspondence should be addressed to H. C. Ananda Murthy; anandkps350@gmail.com
}

Received 2 April 2020; Revised 6 May 2020; Accepted 13 May 2020; Published 1 June 2020

Academic Editor: Valeri P. Tolstoy

Copyright (C) 2020 H. C. Ananda Murthy et al. This is an open access article distributed under the Creative Commons Attribution License, which permits unrestricted use, distribution, and reproduction in any medium, provided the original work is properly cited.

\begin{abstract}
Indigenous medicinal plant of Ethiopia has been applied for the first time to investigate the synergistic influence of phytoconstituents in green copper nanoparticles ( $\mathrm{g}-\mathrm{Cu} \mathrm{NPs}$ ) towards the enhancement of antimicrobial properties of NPs. We report the green synthesis of $\mathrm{Cu}$ NPs using Hagenia abyssinica (Brace) JF. Gmel. leaf extract. The synthesized g-Cu NPs were characterized by UV-visible, UV-DRS, FT-IR, XRD, SEM, EDXA, TEM, HRTEM, and SAED techniques. The maximum absorbance, $\lambda_{\max }$, was found to be $403 \mathrm{~nm}$ for $\mathrm{g}-\mathrm{Cu}$ NPs due to surface plasmon resonance. The energy gap, $E_{\mathrm{g}}$ of NPs, was found to be $2.19 \mathrm{eV}$. FTIR spectra confirmed the presence of polyphenols, tannins, and glycosides in the leaf extract of Hagenia abyssinica. The spectral band at $740 \mathrm{~cm}^{-1}$ is a characteristic of interaction between $\mathrm{Cu}$ and biomolecules of the extract. The XRD analysis revealed that the g-Cu NPs appears to be more crystalline in nature. SEM and TEM micrographs showed a mix of spherical, hexagonal, triangular, cylindrical, and irregularly shaped $\mathrm{Cu}$ particles. The average particle size of NPs was found to be $34.76 \mathrm{~nm}$ by ImageJ analysis. EDX analysis confirmed the presence of copper in the g-Cu NPs. In addition, the SAED pattern of g-Cu NPs presented concentric circular patterns for 4 major planes of crystalline copper and its oxides. The experimental and calculated $d$-spacing values of one of the crystal planes (111) were found to be $0.2432 \mathrm{~nm}$ and $0.2444 \mathrm{~nm}$, respectively. The $d$-spacing values of $0.2444 \mathrm{~nm}$ and $0.2040 \mathrm{~nm}$ correspond to $\mathrm{d}_{111} \mathrm{Cu}_{2} \mathrm{O}$ and $\mathrm{d}_{111} \mathrm{Cu}$ lattice fringes, respectively. The antibacterial test conducted on E. coli, Pseudomonas aeruginosa, Staphylococcus aureus, and Bacillus subtilis showed good zone of inhibitions $12.7,12.7,14.7$, and $14.2 \mathrm{~mm}$, respectively, proving potentiality of g-Cu NPs as a remedy for infectious diseases caused by tested pathogens.
\end{abstract}

\section{Introduction}

Metallic nanoparticles are multifunctional in nature, and they have been extensively used in a variety of sectors of industries and medicine including drug delivery, cancer treatment, wastewater treatment, and DNA analysis, as antibacterial agents and biosensors and in solar power generation and catalysis. The green synthesis of metallic nanoparticles has been proposed as a cost-effective and environmentally friendly alternative to chemical and physical methods. In recent years, copper nanoparticles (Cu NPs) have attracted much attention of researchers due to its applications in industries and medicine. However, other nanoparticles, such as platinum, gold, iron oxide, silicon oxides, and nickel, have not shown bactericidal effects in studies with Escherichia coli [1].

Many plant parts or whole plants have been used for the green synthesis of $\mathrm{Cu}$ NPs due to the presence of a large number of bioactive compounds in plants. The extracts of plants have been efficiently applied for this purpose. Synthesis of 


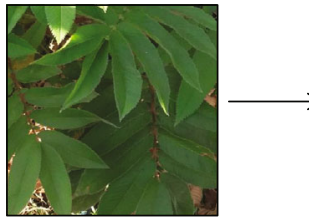

Plant leaves

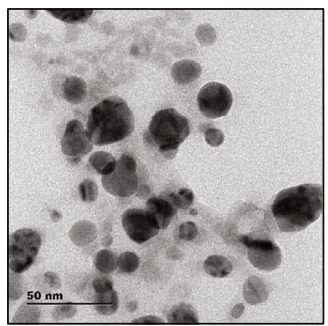

TEM of g-Cu NPs

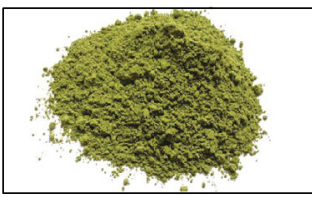

Plant powder

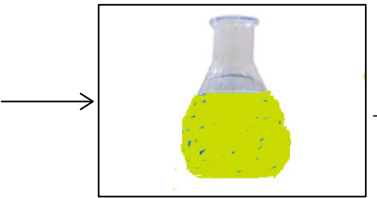

Plant extract

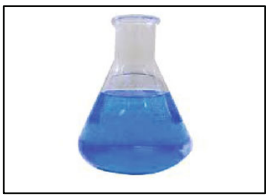

Copper solution

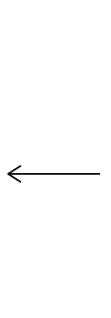

Figure 1: The scheme of synthesis of g-Cu NPs.

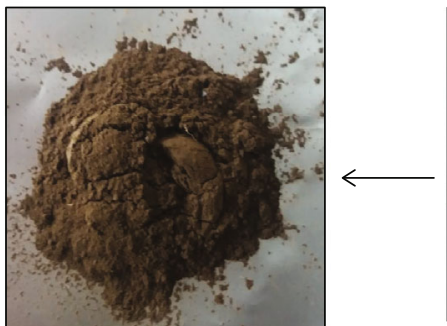

g-Cu NPs

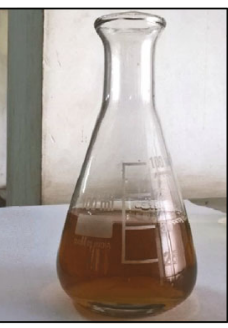

$\mathrm{Cu}$ NPs solution
$\mathrm{Cu}$ NPs has been successful with extracts of various parts of plant species that include Punica granatum peel [2], Zingiber officinale stem [3], Citrus medica Linn. (Idilimbu) juice [4], Ziziphus spina-christi (L.) Willd fruit [5], Asparagus adscendens Roxb. root and leaf [6], Eclipta prostrata leaf [7], Ginkgo biloba Linn leaf [8], Plantago asiatica leaf [9], Thymus vulgaris L [10], black tea leaf [11], Terminalia catappa leaf [12], and Azadirachta indica leaf [13].

The study on antibacterial effects of copper nanoparticles using E. coli and Bacillus subtilis revealed the fact that the $\mathrm{Cu}$ NPs exhibited superior antibacterial activity compared to the silver nanoparticles. Cu NPs demonstrated good antimicrobial influence on Bacillus spp. and prominent fungicidal influence on Penicillium spp. microorganisms. Cu NPs exhibited greater inhibition on Escherichia coli in comparison with Klebsiella pneumoniae, Pseudomonas aeruginosa, Propionibacterium acnes, and Salmonella typhi. Cu NPs have been synthesized by using extracts of various plants found all over the globe [14]. But no research has been conducted as far as green synthesis of $\mathrm{Cu}$ NPs is concerned using extracts of medicinal plants of Ethiopia.

Therefore, the present research work was proposed to explore the synthesis of green $\mathrm{Cu}$ NPs (g-Cu NPs) using extracts of medicinal plant of Ethiopia. More than 95\% of traditional medical preparations in Ethiopia are of plant origin. A medicinal plant species of Ethiopia identified for the biogenic synthesis of g-Cu NPs in aqueous media is Hagenia abyssinica (Brace) JF. Gmel.

Hagenia abyssinica (Brace) JF. Gmel, a member of the Rosaceae family, is a species of flowering plant native to the high-elevation Afromontane regions of central and eastern Africa from Sudan and Ethiopia. It is known in English as African redwood and East African rosewood and in Amharic language as kosso. Hagenia abyssinica is a slender tree up to $20 \mathrm{~m}$ tall, with a short trunk and thick branches. The roots are cooked with meat and the soup drunk for general illness and malaria, while the dried and pounded female inflorescence is
TABle 1: The details of phytoconstituents screening of Hagenia abyssinica (Brace) JF. Gmel. plant leaf extract.

\begin{tabular}{lccc}
\hline Sl. no. & Phytoconstituents & Test/reagent & Result \\
\hline 1 & Alkaloids & Wagner's reagent & - \\
2 & Tannins & $\mathrm{KOH}$ & + \\
3 & Flavonoids & Shinoda test & - \\
4 & Terpenoids & Salkowski test & - \\
5 & Anthraquinone glycosides & Borntrager's test & + \\
6 & Cardiac glycosides & Keller-Kiliani test & + \\
7 & Saponins & Frothing test & - \\
8 & Phenols & FeCl $_{3}$ & + \\
\hline
\end{tabular}

used as an anthelmintic (especially for tapeworm). Bark may be pounded and added to cold water and the liquid drunk as a remedy for diarrhea and stomachache. Generally, this is a strong medicine that must not be taken in large quantities; it is sometimes taken as an abortifacient [15].

The aim of this research work was to synthesize g-Cu NPs mediated by Hagenia abyssinica (Brace) JF. Gmel, medicinal plant leaf extract. The integration of bioactive compounds of this medicinal plant with $\mathrm{Cu}$ NPs is believed to be extremely beneficial for varieties of ailments. This green synthesis method was devised to eliminate the use of toxic chemicals which play roles of reducing agents and capping agents during chemical synthesis. The biogenically synthesized g-Cu NPs were characterized using UV-visible, UVDRS, FT-IR, XRD, SEM, EDXA, TEM, HRTEM and SAED techniques. The antimicrobial properties of $\mathrm{g}-\mathrm{Cu}$ NPs have also been evaluated against gram-positive and gram-negative bacteria. The present research work gives comprehensive report on synthesis and antimicrobial activities of g-Cu NPs which in turn will have significant impact on biomedical applications. 


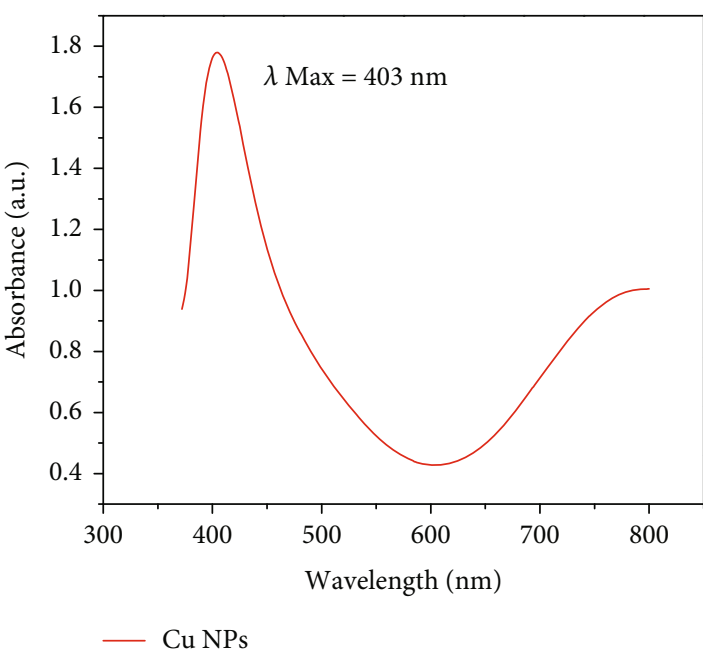

(a)

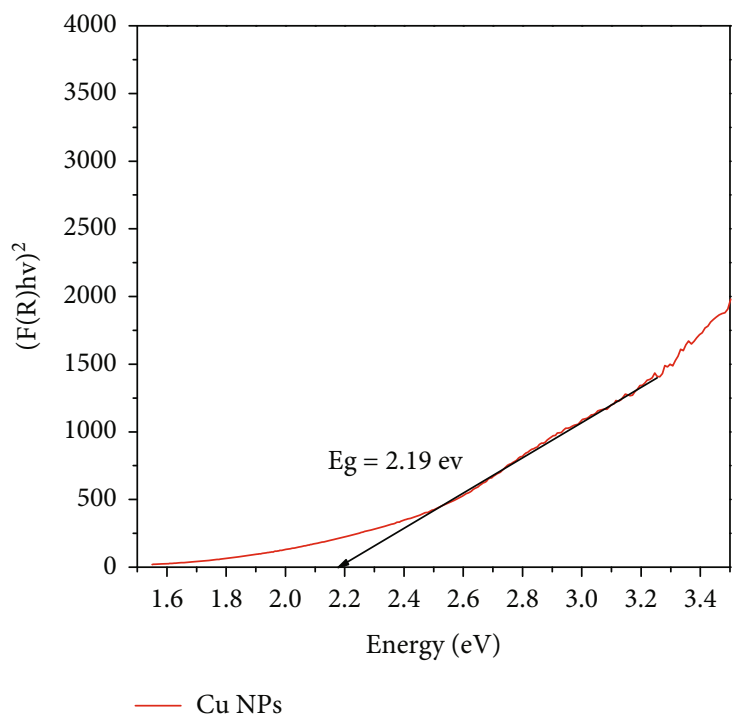

(c)

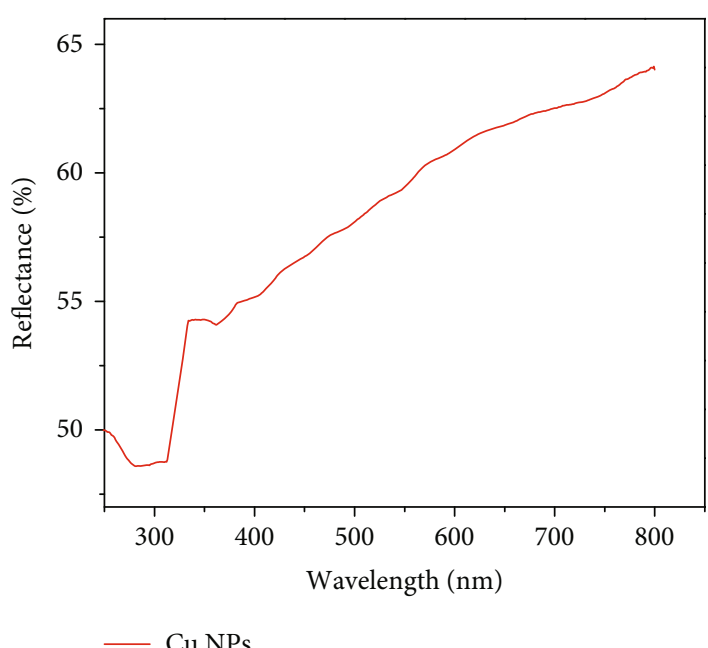

(b)

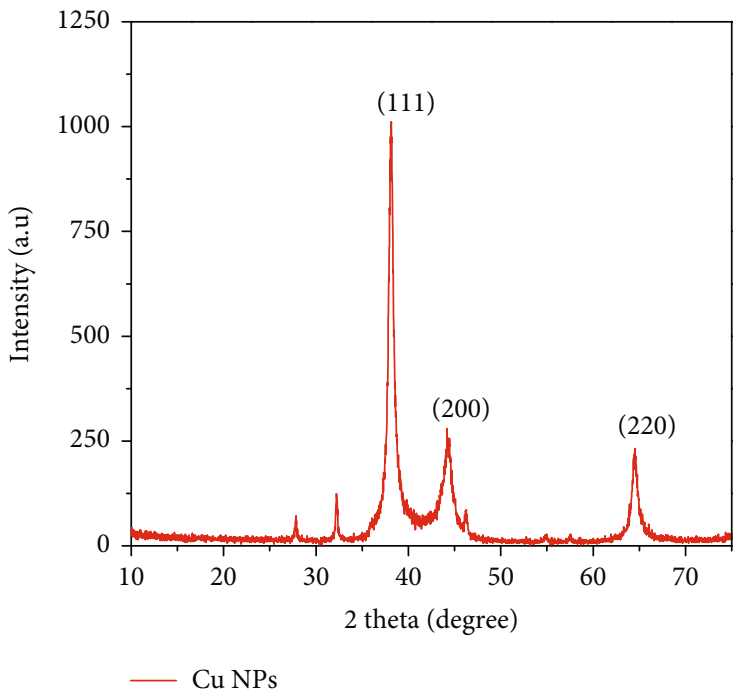

(d)

Figure 2: (a) UV-visible absorbance spectrum of g-Cu NPs. (b) UV-visible diffused reflectance spectrum of g-Cu NPs. (c) Tauc plot of g-Cu NPs showing its $E_{\mathrm{g}}$ value. (d) XRD diffraction pattern of g-Cu NPs.

\section{Materials and Methods}

2.1. Materials and Reagent. All the chemicals $\left(\mathrm{Cu}\left(\mathrm{NO}_{3}\right)_{2} \cdot 3 \mathrm{H}_{2} \mathrm{O}\right.$, ethanol, Muller agar solution, and dimethyl sulfoxide DMSO) used in the experiments were of analytical grade (purchased from Merck chemical Industrial company) and used without any further purification.

2.2. Collection and Authentication of Plant Materials. Hagenia abyssinica (Brace) JF. Gmel. plant leaves were collected from Ethiopian Institute of Agricultural Research, Wondo Genet, Oromia Regional State, Southern Ethiopia, after conducting the field surveys.

The coordinates of the research center are $\mathrm{N} 7^{\circ} 5^{\prime}$ $31.5096^{\prime \prime}$ E $38^{\circ} 37^{\prime} 54.7284^{\prime \prime}$ and $\mathrm{N} 7^{\circ} 5^{\prime} 35.5128^{\prime \prime}$ E $38^{\circ} 37^{\prime}$ $59.0304^{\prime \prime}$. The identity of the plant Hagenia abysinica (Brace) JF. Gmel. (Code EB002) was authenticated by a plant taxonomist at the National Herbarium, Department of
Biology, Addis Ababa University Herbarium, Addis Ababa, Ethiopia.

2.3. Preparation of Plant Leaf Extract. The leaves of Hagenia abyssinica were surface cleaned and washed repeatedly with tap water followed by distilled water to remove dust particles and then allowed to dry under shadow for 15 days to remove moisture contents from the leaves. The dried leaves were ground using a grinding machine followed by packing in a brown bottle. The extraction was carried out by taking $20 \mathrm{~g}$ of the powdered leaves of Hagenia abyssinica in a $500 \mathrm{ml}$ of conical flask containing $400 \mathrm{ml}$ of deionized water. The flask was later covered with aluminum foil, to prevent the effect of light. After that, the mixture was shaken using a mechanical shaker for 90 minutes and allowed to warm at $50^{\circ} \mathrm{C}$ for 1 hour on a magnetic stirrer; then it was allowed to cool down to room temperature overnight. The prepared solution was filtered through Whatman No.1 filter paper to 


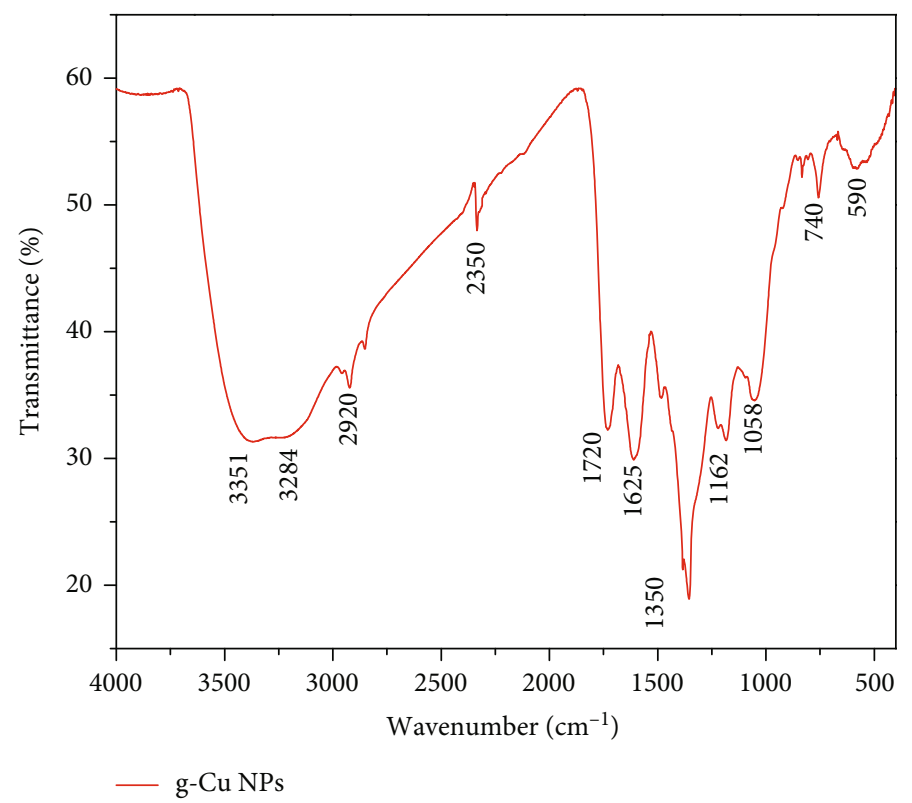

Figure 3: FTIR spectrum of g-Cu NPs.

get clear solution. The filtrate was stored at $4^{\circ} \mathrm{C}$ for future experiments.

2.4. Green Synthesis of $\mathrm{Cu} N P$ s. A $0.2 \mathrm{M}$ aqueous $\mathrm{Cu}\left(\mathrm{NO}_{3}\right)_{2} \cdot 3 \mathrm{H}_{2} \mathrm{O}$ solution was prepared and stored in brown bottles. $100 \mathrm{ml}$ of plant leaf extract was mixed with $400 \mathrm{ml}$ of $0.2 \mathrm{M} \mathrm{Cu}\left(\mathrm{NO}_{3}\right)_{2} \cdot 3 \mathrm{H}_{2} \mathrm{O}$ solution $(1: 4)$ slowly dropwise with constant stirring.

The mixture has been incubated at room temperature for 24 hrs. The color change was checked periodically (after 30 minutes and 60 minutes). The change in color from blue to light brownish visually indicates the formation of $\mathrm{Cu}$ NPs, and then, the solution was centrifuged for $15 \mathrm{~min}$ at $10000 \mathrm{rpm}$. The obtained g-Cu NPs (Figure 1) were washed by deionized water and ethanol to remove any impurities. Thereafter, the NPs were allowed to dry and ground so as to be used for further analysis.

2.5. Characterization Techniques. The UV-visible absorbance and reflectance spectra of the samples were recorded in the range of 200-800 nm using Shimadzu's UV-2600, UVvisible spectrophotometer. UV-visible spectra were measured by transmission, whereas UV-DRS specifically refers to diffuse reflection spectroscopy. The $\mathrm{Cu}$ nanoparticle solution was prepared by mixing the freshly prepared plant extract solution with $\mathrm{Cu}$ solution after appropriate dilution. An absorbance spectrum was recorded by allowing the instrument to scan wavelengths ranging between 200 and $800 \mathrm{~nm}$, and the absorbance of the nanoparticle solution against blank extract solution was collected. Fourier transform-infrared spectroscopy (FT-IR) spectrum (65 FTIR PerkinElmer) was recorded using $\mathrm{KBr}$ pellets in the range of $400-4000 \mathrm{~cm}^{-1}$ [16]. X-ray diffraction (XRD-Shimadzu $\mathrm{X}$-ray diffractometer) (PXRD-7000) analytical technique was used to reveal the crystalline nature of $\mathrm{g}$ - $\mathrm{Cu}$ NPs. The scanning electron microscopy with energy-dispersive X-ray spectroscopy (SEM-EDX-EVO 18 model with low vacuum facility and ALTO 1000 Cryo attachment) and transmission electron microscope with high-resolution (JEOL JEM 2100 HRTEM) were used for understanding morphological and structural features of g-Cu NPs. Gatan Digital Micrograph Software was used to evaluate $d$-spacing values of lattice fringes. Particle size was computed by using ImageJ application.

2.6. Method of Antimicrobial Evaluation. The in vitro antibacterial activity of g-Cu NPs was evaluated using an Agar disc-diffusion method [17] against selected two grampositive bacteria (Staphylococcus aureus and Bacillus subtilis) and two gram-negative pathogenic bacteria (Escherichia coli and Pseudomonas aeruginosa). Microbial strains were obtained from pastor institute. Prior to an antibacterial activity test, the bacterial strains were cultured in nutrient broth for 24 hours to obtain logarithmic growth phase of the test bacteria. The actively growing bacterial cultures of $1.3 \times 10^{8}$ $\mathrm{CFU} / \mathrm{ml}$ concentration were inoculated/spread into the Muller Hinton Agar (MHA) [18]. The extract was prepared at $1 \mathrm{mg} / \mathrm{ml}$ (dissolved in Dimethyl Sulfoxide). The Whatman filter paper disks were punched at $6 \mathrm{~mm}$ diameter and impregnated with the dissolved extract and then placed to the MHA surface. Ampicillin disc was used as a positive control while DMSO was taken as the negative control. The plates were incubated at $37^{\circ} \mathrm{C}$ for 24 hours. The antimicrobial activity was evaluated in terms of zone of inhibition, measured, and recorded in millimeters using a ruler.

\section{Results and Discussion}

3.1. Synthesis of $g$-Cu NPs. The g-Cu NPs were synthesized by using copper nitrate as a precursor and plant leaf extract as a reducing and capping agent. The change in color from blue to light brownish visually indicates the formation of copper 


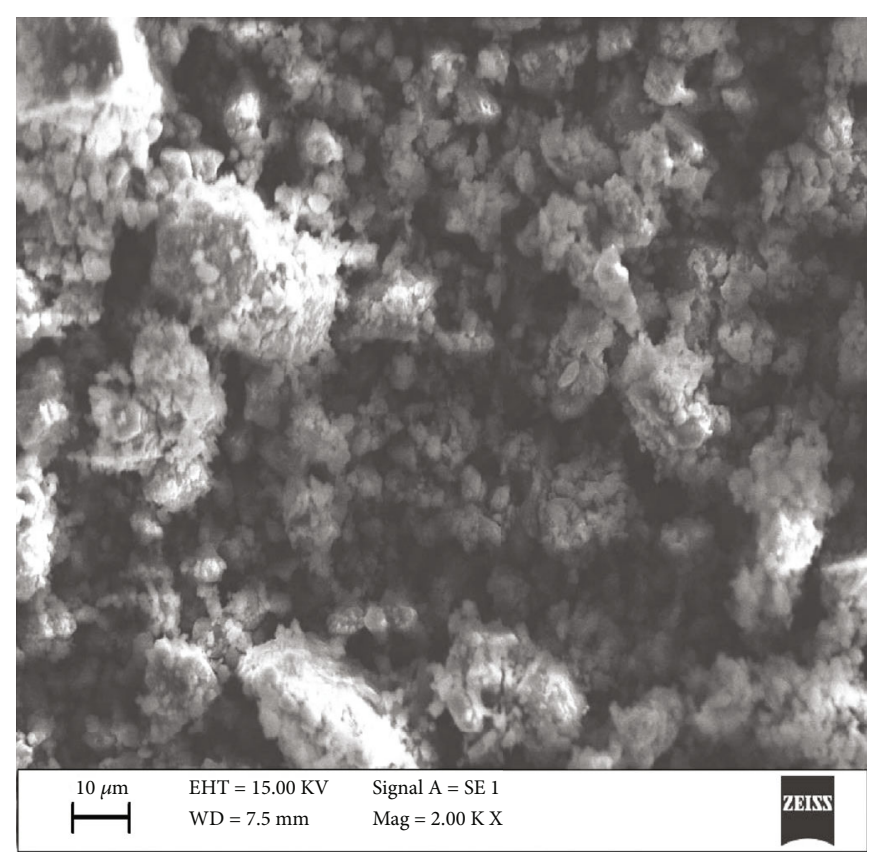

(a)

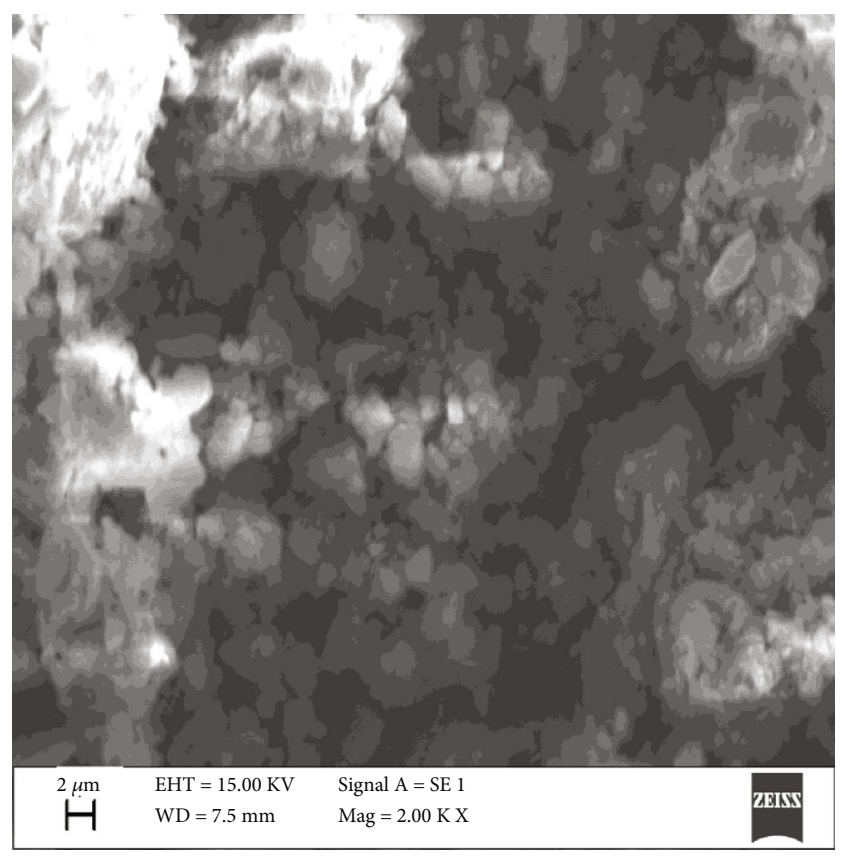

(b)

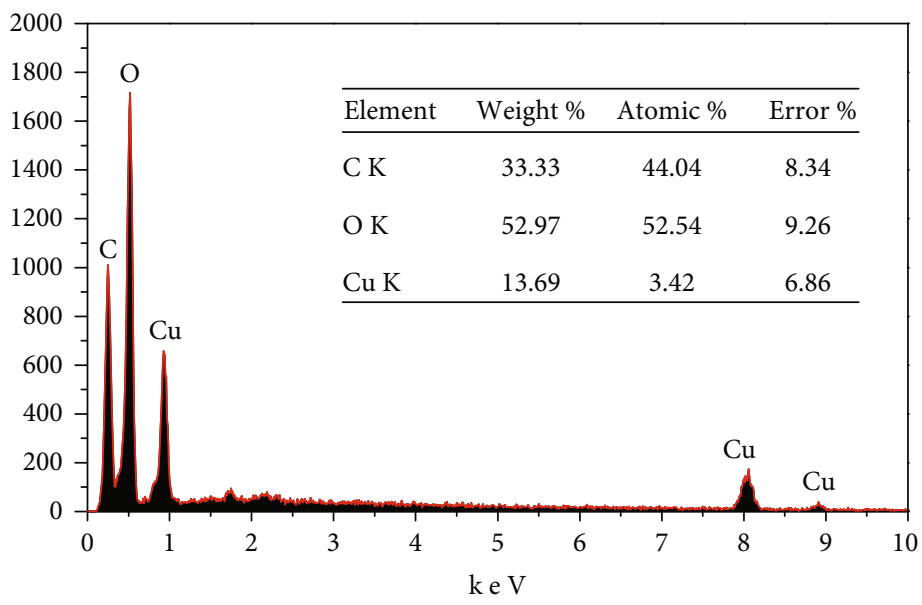

(c)

Figure 4: (a, b) SEM micrographs of g-Cu NPs. (c) EDAX spectrum of g-Cu NPs.

NPs. The g-Cu NPs were washed by deionized water and ethanol to remove any unwanted particles. Thereafter, g-Cu NPs were dried and ground (Figure 1) and later subjected to various characterization methods.

The presence of phenolic compounds, tannins, and different glycosides was confirmed during the phytochemical screening of Hagenia abyssinica (Brace) JF. Gmel. leaf extract. The details of the screening are as given in Table 1. It is possibly believed that the bioactive compounds such as phenolic compounds act as a ligand and bind to metal ions and reduce them and cap them to form nanoparticles. These ligands also act as particle size controllers as reported by the earlier researcher [19].

Primary components of Hagenia abyssinica extract are phenolic compounds, tannins, anthraquinone glycosides, and cardiac glycosides [15]. The antioxidant properties of polyphenolic compounds are primarily due to their high inclination towards chelating the metals. Phenolic compounds contain hydroxyl and carboxylic groups which have very high tendency to bind metal ions. Metal ions in solution interact with polyphenolic compound and helps in the nucleation and formation of $\mathrm{Cu}$ NPs [20].

3.2. Characterization of $\mathrm{g}-\mathrm{Cu}$ NPs. The g-Cu NPs were characterized using UV-visible, UV-DRS, FT-IR, XRD, SEM, EDXA, TEM, HRTEM, and SAED techniques.

3.2.1. UV-Visible Spectral Analysis. The UV-visible absorbance spectrum recorded for g-Cu NPs exhibited $\lambda_{\max }$ of 403 as shown in Figure 2(a) [21].

This absorption band is basically due to surface plasmon resonance of g-Cu NPs. A similar result was reported after the analysis of synthesized $\mathrm{Cu}$ NPs using the Ocimum sanctum leaf extract [22]. The surface plasmon absorbance 


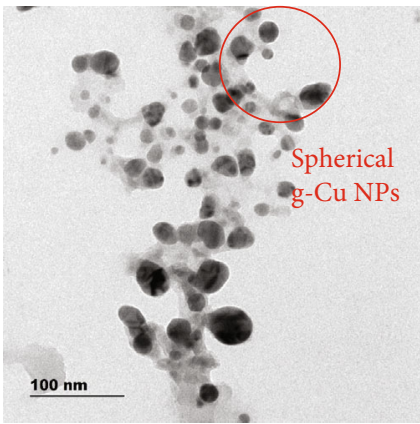

(a)

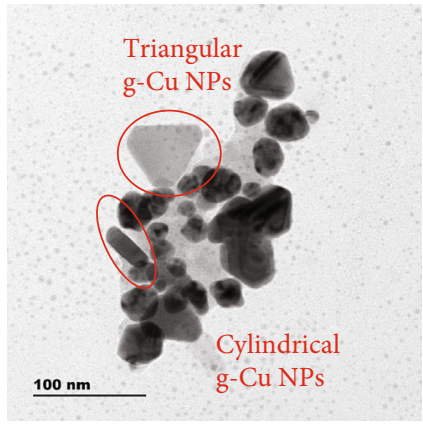

(c)

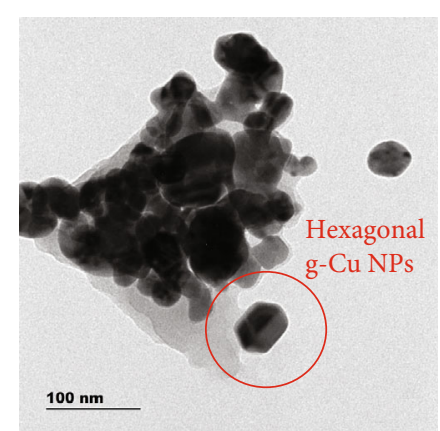

(b)

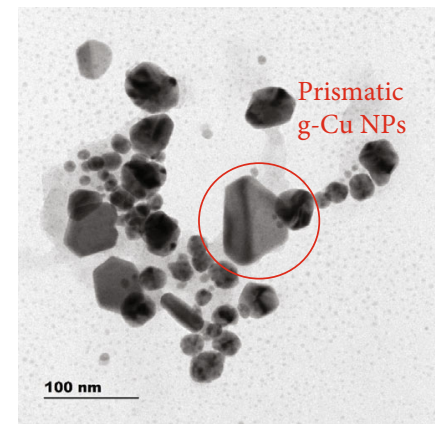

(d)

FIgURE 5: The TEM micrographs showing g-Cu NPs with (a) spherical, (b) hexagonal, (c) triangular and cylindrical, and (d) prismatic shapes.

basically depends on the size of NPs, and hence, every researcher reports a different $\lambda_{\max }$ value for NPs synthesized using different plant extracts. Similarly, the UV-visible diffused reflectance spectrum was recorded (Figure 2(b)). The band gap energy of g-Cu NPs was evaluated using Tauc plot as shown in Figure 2(c) by using the data obtained in reflectance spectra utilizing Kubelka-Munk function. The band gap energy, $E_{\mathrm{g}}$, of g-Cu NPs was found to be $2.19 \mathrm{eV}$.

3.2.2. XRD Analysis. The XRD diffraction pattern of $\mathrm{g}-\mathrm{Cu}$ NPs is presented in Figure 2(d). The peaks observed in the pattern at $2 \theta$ values $=35.76^{\circ}, 44.68^{\circ}$, and $64.37^{\circ}$ corresponds to (111), (200), and (220) lattice planes of face centered cubic structure of $\mathrm{Cu} \mathrm{NPs}$, and the diffraction data were in good agreement with ICSD file no. 04-0836 [23].

3.2.3. FT-IR Spectral Analysis. The FTIR spectra of g-Cu NPs showed peaks at 3351, 3284, 2920, 2350,1720, 1625, 1342, $1160,1058,740$, and $590 \mathrm{~cm}^{-1}$ (Figure 3). From those indicated peaks, the broad absorption peaks of g-Cu NPs observed at $3351 \mathrm{~cm}^{-1}$ and $3254 \mathrm{~cm}^{-1}$ represent the presence of higher concentration of alcohols, with $\mathrm{O}-\mathrm{H}$ stretches and $\mathrm{N}-\mathrm{H}$ stretches of amines.

The intense peaks shown at $2929.0 \mathrm{~cm}^{-1}$ and $2890.6 \mathrm{~cm}^{-1}$ correspond to asymmetric $\mathrm{C}-\mathrm{H}$ stretching of the $-\mathrm{CH}_{2}$ and $\mathrm{CH}_{3}$ groups, respectively. The presence of atmospheric $\mathrm{CO}_{2}$ resulted in a stretching vibration located at a wave number of $2350 \mathrm{~cm}^{-1}$. It is also believed that the amine and carboxylate group present in the leaf extract of Hagenia abyssinica is responsible for the binding of proteins with the surface of $\mathrm{Cu}$ and thereby leading to the stabilization of the biosynthesized nanoparticles.
The absorption peaks represented at $1720 \mathrm{~cm}^{-1}$ and $1625 \mathrm{~cm}^{-1}$ indicate the presence of $\mathrm{C}=\mathrm{O}$ and $\mathrm{C}=\mathrm{C}$ bending modes of vibrations. The peaks represented by $1350 \mathrm{~cm}^{-1}$ show $\mathrm{C}-\mathrm{N}$ stretching of amide. The medium peak at $1160 \mathrm{~cm}^{-1}$ corresponds to C-O stretching of phenolic compounds. The $\mathrm{C}-\mathrm{O}-\mathrm{C}$ stretching appears at $1058 \mathrm{~cm}^{-1}$. The last peak at 590 corresponds to bending modes of vibrations of the $\mathrm{C}-\mathrm{H}$ bond. FTIR analysis results confirmed the presence of various phytochemicals of Hagenia abyssinica (Brace) JF. $G m e l$. leaf extract such as phenolics, tannins, and proteins involved in the synthesis of g-Cu NPs [24]. In addition to this, the FTIR spectrum of $\mathrm{Cu}$ NPs shows peaks corresponding to the broad band centered at $740 \mathrm{~cm}^{-1}$ which represents a characteristic peak believed to be due to the interaction of $\mathrm{Cu}$ with biomolecules of extract.

3.2.4. Morphological and Compositional Analysis by SEM$E D A X$. The visual displays of size and shape of synthesized g-Cu NPs as depicted by SEM micrographs are shown in Figures 4(a) and 4(b) [25]. The SEM images also demonstrated the nonhomogeneity of the particles in terms of their shape and size. All the possible spherical and irregular shapes such as truncated hexagonal, cylindrical, triangular, and prismatic shapes of $\mathrm{Cu}$ NPs with varying particle sizes were found in the micrographs [26]. The average grain size of $\mathrm{Cu}$ NPs was found to be in the range of $10-50 \mathrm{~nm}$. It seems particles were found to agglomerate moderately due to a high surface area resulting in the formation of medium-sized particles. The chemical composition of the NPs was studied by EDAX analysis. Figure 4(c) shows the EDAX spectrum obtained for the g-Cu NPs. The peaks corresponding to elemental $\mathrm{Cu}, \mathrm{C}$, and $\mathrm{O}$ were clearly identified, and no 


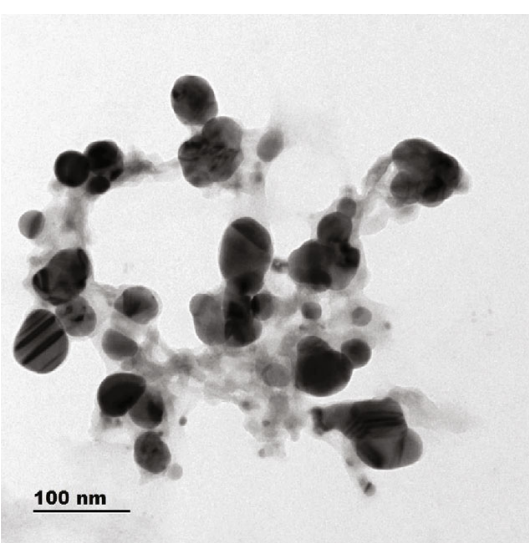

(a)

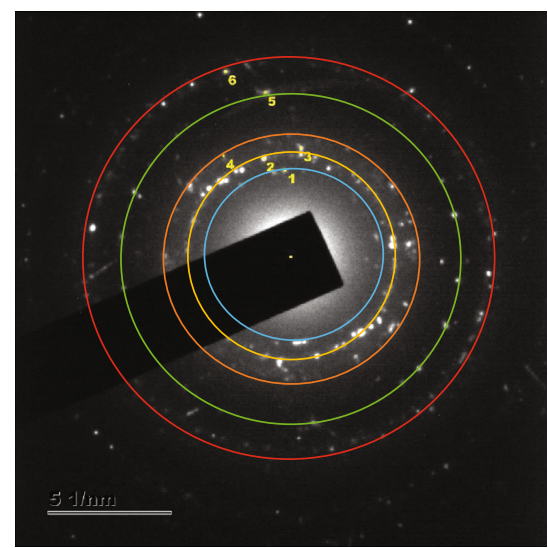

(c)

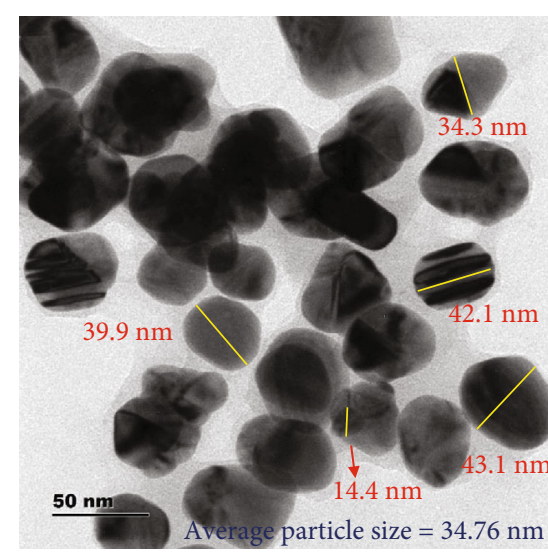

(b)

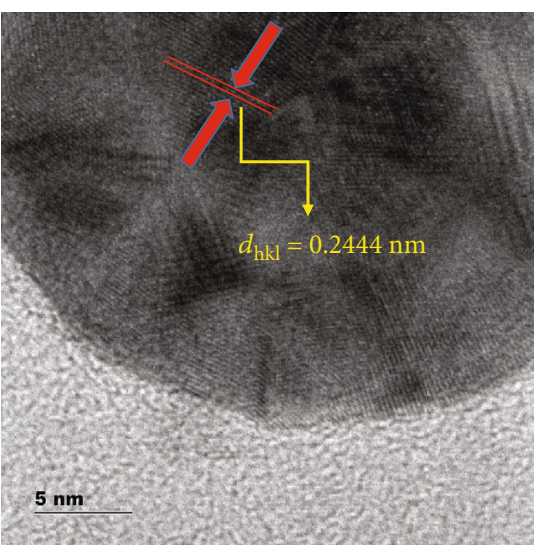

(d)

FIgURE 6: TEM images of as-synthesized g-Cu NPs at (a) lower magnification (100 nm) and (b) higher magnification (50 nm). (c) SAED pattern with 1 to 6 spots and (d) HRTEM image showing lattice fringes of g-Cu NPs with $d$-spacing of $0.2444 \mathrm{~nm}$.

additional peaks were present, which demonstrates the purity of the synthesized NPs, and this was consistent with the XRD studies.

It is also possibly believed that the presence of $\mathrm{C}$ and $\mathrm{O}$ is basically from the capped bioactive compounds. In addition, it is also expected that very few copper atoms at the surface of the NPs might have been oxidized to yield small amounts of $\mathrm{CuO}$ and $\mathrm{Cu}_{2} \mathrm{O}$. The reduction of copper ions to $\mathrm{Cu}$ NPs is facilitated by the biomolecules of plant extract containing surface hydroxyl groups.

3.2.5. TEM, HRTEM, and SAED Analysis. To get the further deep insight on the morphology, size, and crystalline nature of the g-Cu NPs, TEM, HRTEM, and SAED analysis was employed. The HRTEM images of as-synthesized g-Cu NPs (Figure 5) shows that the synthesized NPs are mostly spherical but exhibited different shapes. Smaller NPs as small as $10 \mathrm{~nm}$ confirm the efficient role of bioactive components of Hagenia abyssinica (Brace) JF. Gmel. plant extract as capping and stabilizing agents. Otherwise, NPs would have been agglomerated to yield larger NPs in an elongated form [5].

In addition, the variation in size of $\mathrm{g}-\mathrm{Cu}$ NPs is probably due to the presence of polyphenolic compounds (from Hagenia abyssinica (Brace) JF. Gmel, leaf extract) which have strong attractive forces and holds the particles together.
The TEM micrographs which exhibited very finely grained g-Cu NPs with spherical, cylindrical, prismatic, hexagonal, triangular, and near spherical shapes are presented in Figures 5(a)-5(d).

All these near spherical particles with varying sizes from $14.4 \mathrm{~nm}$ to $43.1 \mathrm{~nm}$ with an average particle size of $34.76 \mathrm{~nm}$ as determined by Image J application are as shown in Figures 6(a) and 6(b).

The SAED pattern of g-Cu NPs (Figure 6(c)) contained six spots each corresponding to specific crystal planes. One of such planes is presented with a $d$-spacing of $0.2444 \mathrm{~nm}$ as shown in Figure 6(d).

HRTEM morphologies of g-Cu NPs with magnified lattice fringes, IFFT patterns, and profile of IFFT with a $d$ -spacing value for a specified plane (Figure 6(d)) are presented in Figures $7(\mathrm{a})-7(\mathrm{c})$, respectively. The analysis to arrive at a $d$-spacing value has been carried out by using Gatan Digital Micrograph Software application which resulted in a $d_{\mathrm{hkl}}$ value of $0.2444 \mathrm{~nm}$ for a set of crystal planes at the surface of $\mathrm{Cu}$ NPs.

The $d$-spacing values for all the spots depicted in the SAED pattern of g-Cu NPs (Figure 6(c)) are presented in Table 2. Each spot on the SAED pattern corresponds to a specific set of lattice planes. The XRD pattern of g-Cu NPs presented earlier in Figure 2(d) revealed 3 major peaks 


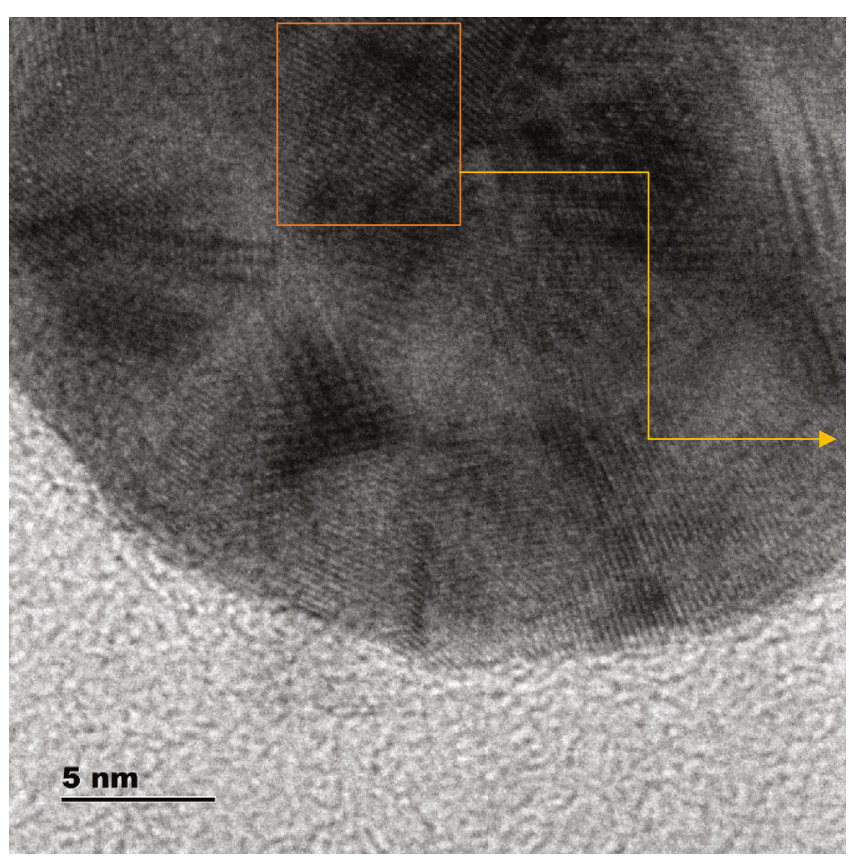

(a)

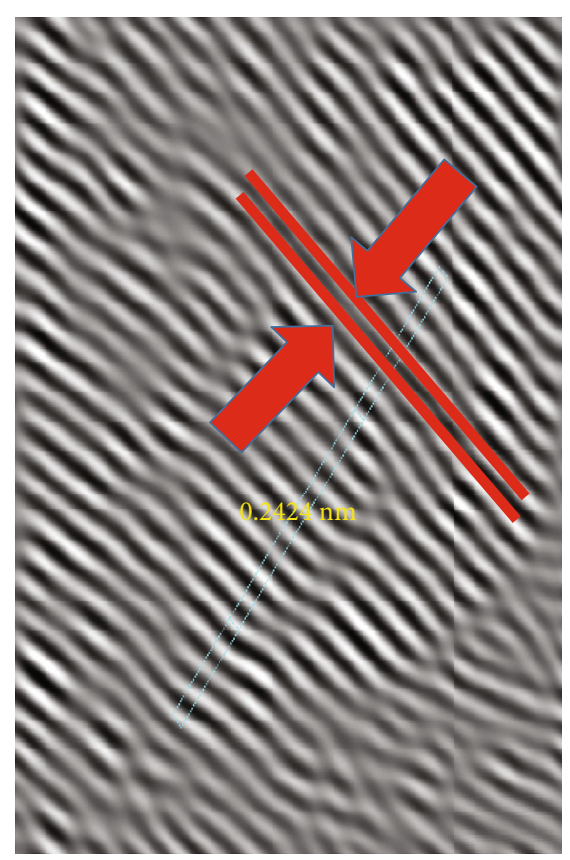

(b)

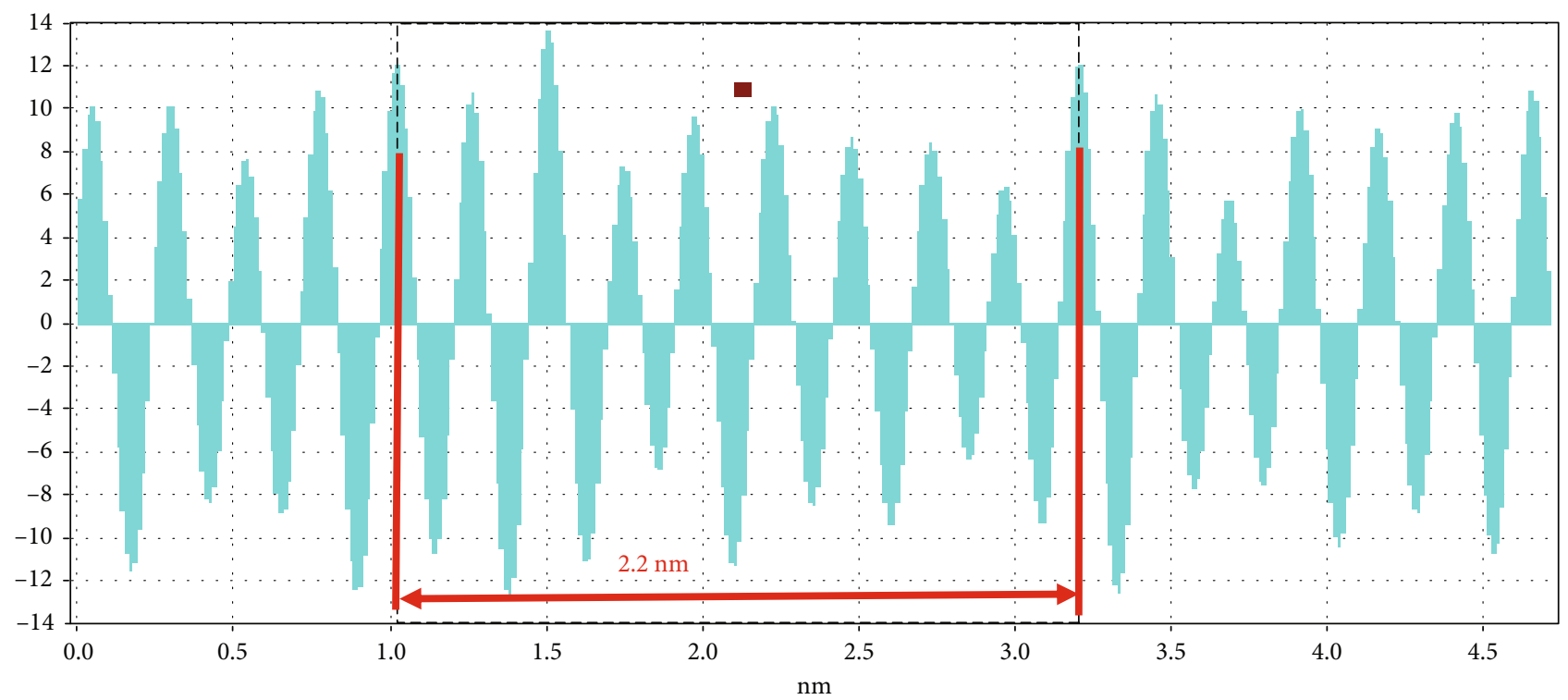

(c)

FIGURE 7: HRTEM morphology of g-Cu NPs (a), magnified lattice fringes (b), and IFFT patterns (c). Profile of IFFT with $d$-spacing distance.

TABLE 2: The $d$-spacing values for g-Cu NPs from the SAED pattern.

\begin{tabular}{|c|c|c|c|c|c|}
\hline Spot no. & $d$-spacing $(\mathrm{nm})$ & Rec. Pos. $(1 / \mathrm{nm})$ & Degrees to spot 1 & Degrees to $x$-axis & Amplitude \\
\hline 1 & 0.2854 & 3.504 & 0.00 & 93.82 & 284.20 \\
\hline 2 & 0.2432 & 4.112 & 11.68 & 105.50 & 1093.93 \\
\hline 3 & 0.2271 & 4.404 & 9.20 & 84.62 & 612.11 \\
\hline 4 & 0.2040 & 4.903 & 30.11 & 123.93 & 178.68 \\
\hline 5 & 0.1491 & 6.707 & 5.14 & 98.96 & 316.22 \\
\hline 6 & 0.1261 & 7.928 & 15.73 & 109.55 & 440.04 \\
\hline
\end{tabular}


TABLE 3: The variation of zone of inhibitions for different bacterial pathogens by g-Cu NPs.

\begin{tabular}{lcccc}
\hline Sample & E. coli & \multicolumn{2}{c}{ Zone of inhibition (ZI/mm) } \\
& $(0.5 \mathrm{McFarland})$ & $\begin{array}{c}\text { Staphylococcus aureus } \\
(0.5 \mathrm{McFarland})\end{array}$ & $\begin{array}{c}\text { Bacillus subtilis } \\
(0.5 \mathrm{McFarland})\end{array}$ & $\begin{array}{c}\text { Pseudomonas aeruginosa } \\
(0.5 \mathrm{McFarland})\end{array}$ \\
\hline g-Cu NPs & $12.7 \pm 0.4$ & $14.7 \pm 0.2$ & $14.2 \pm 0.8$ & $12.7 \pm 1.1$ \\
Ampicillin disc (+ve control) & 15 & 16 & 16 & 15 \\
\hline
\end{tabular}

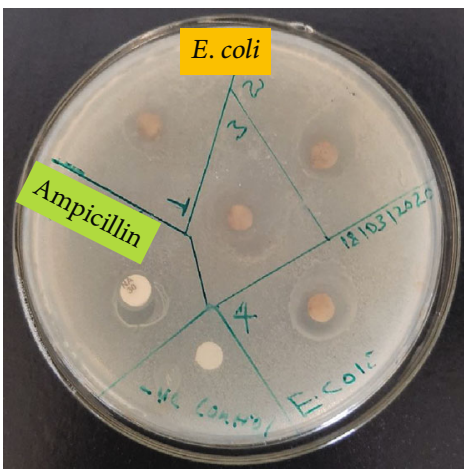

(a)

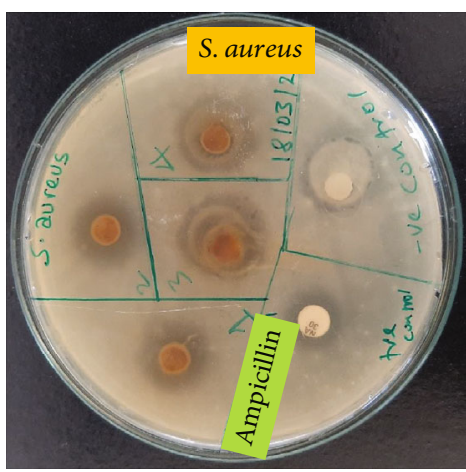

(c)

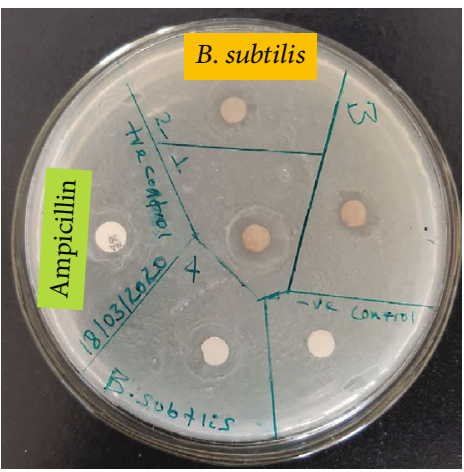

(b)

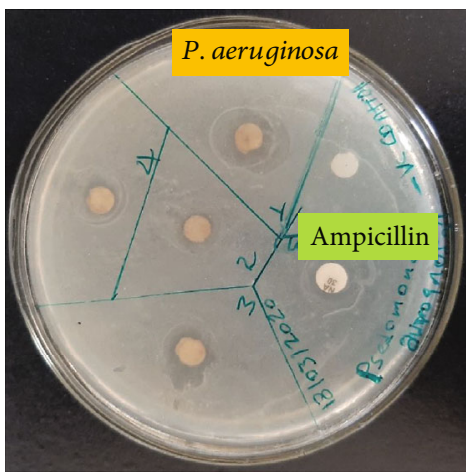

(d)

Figure 8: The antibacterial activity of g-Cu NPs towards pathogens (a) E. coli, (b) B. subtilis, (c) S. aureus, and (d) P. aeruginosa.

corresponding to (111), (200), and (220) planes of the fcc structure of pure $\mathrm{Cu}$ (ICSD no. 04-0836). The $d$-spacing values of the derived diffraction planes from spot 1 to spot 6: $d_{-111} \mathrm{CuO}=0.2854 \mathrm{~nm}, d_{111} \mathrm{Cu}_{2} \mathrm{O}=0.2432 \mathrm{~nm}, d_{200} \mathrm{Cu}=$ $0.2271 \mathrm{~nm}, \quad d_{111} \mathrm{Cu}=0.2040 \mathrm{~nm}, d_{-111} \mathrm{Cu}_{2} \mathrm{O}=0.1491 \mathrm{~nm}$, and $d_{220} \mathrm{Cu}=0.1261 \mathrm{~nm}$, are in agreement with the $d$-spacing values of $\mathrm{CuO}$ (ICSD no. 48-1548, tenorite-C2/c), $\mathrm{Cu}_{2} \mathrm{O}$ (ICSD no. 05-0667, cuprite-Pn-3 m), and Cu (ICSD no. 04-0836) structures. The similar result was reported in the recent work [27].

The estimated $d$-spacing value of lattice fringes at the surface of the Cu NPs is $0.2444 \mathrm{~nm}$, which is comparable to the $d_{\text {hkl }}$ value of the (111) plane of fcc-structured $\mathrm{Cu}_{2} \mathrm{O}$. It can be concluded that few copper atoms at the surface of NPs possibly reacted with air to form their oxides, $\mathrm{Cu}_{2} \mathrm{O}$ and $\mathrm{CuO}$.

3.3. Antimicrobial Activity. The g-Cu NPs synthesized by using the leaf extract of Hagenia abyssinica (Brace) JF. Gmel. showed a broad range of antibacterial activities against all tested pathogens: E. coli, Pseudomonas aeruginosa, Staphylococcus aureus, and Bacillus subtilis with a zone of inhibition $12.7,12.7,14.7$, and $14.2 \mathrm{~mm}$, respectively (Table 3 ). The $\mathrm{Cu}$ NPs have proven record of an excellent antimicrobial activity [28]. Accordingly, this result showed that the g-Cu NPs are more active against gram-positive bacteria than gram-negative bacteria which is possibly due to the difference in the structure of cell walls of bacteria. The antimicrobial activity of NPs can be attributed to the presence of bioactive compounds on the surface of NPs as capping and stabilizing agents. In this regard, the activity was pronounceable against S. aureus compared to ampicillin, the standard antibiotic. The highest zone of inhibition $(\mathrm{mm})$ recorded with $\mathrm{g}-\mathrm{Cu}$ NPs against $S$. aureus bacteria was $14.7 \mathrm{~mm}$, and the lowest zone of inhibition $(\mathrm{mm})$ recorded against $P$. aeruginosa bacteria was $12.7 \mathrm{~mm}$. The wide zone of inhibitions (Figure 8) of g-Cu NPs against pathogens confirms their great potential as a remedy for infectious diseases caused by the tested bacterial pathogens. 
TABLE 4: Comparative statistics of antimicrobial activities of $\mathrm{Cu}$ NPs synthesized by using various plant and algal extracts.

\begin{tabular}{lcccc}
\hline Sl. no. & Plant extract & Zone of inhibition $(\mathrm{mm})$ & Tested pathogens & Reference \\
\hline 1 & Syzygium aromaticum bud extract & 7 & E. coli & {$[23]$} \\
2 & S. lavandulifolia flower extract & 12 & P. aeruginosa & {$[19]$} \\
3 & Ziziphus spina-christi (L.) Willd. fruit extract & 13 & E. coli & {$[5]$} \\
4 & Green and black tea leaf extract & 14 & S. aureus & {$[11]$} \\
5 & Black grape leaf extract & 14 & S. aureus & {$[29]$} \\
6 & Hagenia abyssinica (Brace) JF. Gmel. leaf extract & 14.7 & E.coli & Present work \\
7 & Green alga Botryococcus braunii extract & 17 & P. aeruginosa & {$[26]$} \\
8 & Punica granatum peel extract & 18.67 & P. aeruginosa & {$[2]$} \\
\hline
\end{tabular}

Additionally, the standard disc ampicillin showed the comparable zone of inhibition with the g-Cu NPs, which is small, and it can be attributed to the development of resistance by bacteria against ampicillin.

The antimicrobial results obtained using g-Cu NPs were found to be better when compared with an earlier work reported by many researchers (Table 4). The highest zone of inhibition $(\mathrm{mm})$ recorded with $\mathrm{g}$-Cu NPs against bacteria was $14.7 \mathrm{~mm}$ which is far above the zone of inhibition exhibited by $\mathrm{Cu}$ NPs synthesized by various plant extracts as mentioned in Table 4. The last two plant extracts were proved to exhibit a higher zone of inhibition which can be attributed to the presence of different type of phytoconstituents. Thus, it can be concluded that the synergistic effect of $\mathrm{Cu}$ NPs coupled with bioactive compounds such as tannins and glycoside of Hagenia abyssinica (Brace) $J F$. Gmel. leaf extract was proved to be beneficial against pathogens.

Many mechanisms of antibacterial activity have been reported by the past researchers; accordingly, the action of $\mathrm{g}$-Cu NPs on the bacteria is yet to be explored fully. It is assumed that the g-Cu NPs get adsorbed on to the cell wall of bacteria and interacts with the electronegative elements within the cell membrane. This results in failed metabolism thereby leading to interference and disruption of transcription in bacteria and hence causes antibacterial activity by g$\mathrm{Cu}$ NPs. It is also believed that the synergistic effect of $\mathrm{g}-\mathrm{Cu}$ NPs with bioactive compounds of extract would have played significant influence to inhibit the activity of pathogenic bacteria as suggested by the recent researcher [29].

It is possibly understood that the helical structure of DNA molecules would have been disrupted by the action of $\mathrm{Cu}$ NPs. In addition, electrochemical potential across the cell membrane decreases up on the interaction with the released $\mathrm{Cu}$ metal ion by $\mathrm{Cu}$ NPs affecting integrity of the membrane.

\section{Conclusion}

The green copper nanoparticles (g-Cu NPs) were successfully synthesized by using medicinal plant Hagenia abyssinica (Brace) JF. Gmel. leaf extract. The presence of phytoconstituents such as polyphenols of tannins played roles in reducing and capping agents during the formation of g-Cu NPs. The
UV-visible absorbance and reflectance spectra showing $\lambda_{\max }$ of $403 \mathrm{~nm}$ and $E_{\mathrm{g}}$ of $2.19 \mathrm{eV}$, respectively, confirmed the formation of g-Cu NPs. FTIR spectra supported the presence of capping agents on the surface of g-Cu NPs. The crystalline nature and composition of g-Cu NPs were confirmed by XRD pattern and EDX spectrum, respectively. The SEM and TEM micrographs provided enough evidence towards the nanomorphology of g-Cu NPs with all possible shapes including spherical, triangular, hexagonal, and cylindrical shapes with an average particle size of $34.76 \mathrm{~nm}$. HRTEM micrographs and SAED patterns confirmed the presence of $\mathrm{Cu}$ and surface oxidized $\mathrm{Cu}_{2} \mathrm{O}$ NPs. The wide zone of inhibition of g-Cu NPs against pathogens confirms their great potential as a remedy for infectious diseases caused by the tested bacterial pathogens. Finally, it can be concluded that the synergistic effect of bioactive compounds from medicinal plant coupled with $\mathrm{Cu}$ NPs has been proved to be beneficial against pathogens.

\section{Data Availability}

The [Excel] data used to support the findings of this study are available from the corresponding author upon request.

\section{Conflicts of Interest}

The authors declare that they have no conflicts of interest.

\section{Authors' Contributions}

The concept and experiments were designed, conducted, and manuscript written by H C Ananda Murthy. Characterization of samples was done by Mebratu Kassa and Buzuayehu Abebe. Tegene Desalegn participated in compiling the manuscript. Antimicrobial assay was conducted by Temesgen Assefa. All authors read and approved the final manuscript.

\section{Acknowledgments}

The authors gratefully acknowledge Adama Science and Technology University for the financial support and laboratory facility to conduct this research work. This work has been funded by the project (ANSD/04/0453/11-2018) approved by 
the Research and Technology Transfer Office, sanctioned by Adama Science and Technology University, Ethiopia.

\section{References}

[1] A. K. Mittal, Y. Chisti, and U. C. Banerjee, "Synthesis of metallic nanoparticles using plant extracts," Biotechnology Advances, vol. 31, no. 2, pp. 346-356, 2013.

[2] P. Kaur, R. Thakur, and A. Chaudhury, "Biogenesis of copper nanoparticles using peel extract of Punica granatum and their antimicrobial activity against opportunistic pathogens," Green Chemistry Letters and Reviews, vol. 9, no. 1, pp. 33-38, 2016.

[3] M. T. Delma and M. Jaya Rajan, "Green synthesis of copper and lead nanoparticles using ZingiberOfficinale stemextract," International Journal of Scientific and Research Publications, vol. 6, no. 11, pp. 134-137, 2016.

[4] S. Shende, A. P. Ingle, A. Gade, and M. Rai, "Green synthesis of copper nanoparticles by Citrus medica Linn. (Idilimbu) juice and its antimicrobial activity," World Journal of Microbiology and Biotechnology, vol. 31, no. 6, pp. 865-873, 2015.

[5] R. Khani, B. Roostaei, G. Bagherzade, and M. Moudi, "Green synthesis of copper nanoparticles by fruit extract of Ziziphus spina-christi (L.) Willd.: application for adsorption of triphenylmethane dye and antibacterial assay," Journal of Molecular Liquids, vol. 255, pp. 541-549, 2018.

[6] S. Thakur, S. Sharma, S. Thakur, and R. Rai, "Green synthesis of copper nano-particles using Asparagus adscendens Roxb. root and leaf extract and their antimicrobial activities," International Journal of Current Microbiology and Applied Sciences, vol. 7, no. 4, pp. 683-694, 2018.

[7] I. Chung, A. Abdul Rahuman, S. Marimuthu et al., "Green synthesis of copper nanoparticles using Eclipta prostrata leaves extract and their antioxidant and cytotoxic activities," Experimental and Therapeutic Medicine, vol. 14, no. 1, pp. 18-24, 2017.

[8] M. Nasrollahzadeh and S. Mohammad Sajadi, "Green synthesis of copper nanoparticles using Ginkgo biloba L. leaf extract and their catalytic activity for the Huisgen $[3+2]$ cycloaddition of azides and alkynes at room temperature," Journal of Colloid and Interface Science, vol. 457, pp. 141-147, 2015.

[9] M. Nasrollahzadeh, S. S. Momeni, and S. M. Sajadi, "Green synthesis of copper nanoparticles using Plantago asiatica leaf extract and their application for the cyanation of aldehydes using $\mathrm{K}_{4} \mathrm{Fe}(\mathrm{CN})_{6}$," Journal of Colloid and Interface Science, vol. 506, pp. 471-477, 2017.

[10] Z. Issaabadi, M. Nasrollahzadeh, and S. M. Sajadi, "Green synthesis of the copper nanoparticles supported on bentonite and investigation of its catalytic activity," Journal of Cleaner Production, vol. 142, pp. 3584-3591, 2017.

[11] M. A. Asghar, E. Zahir, S. M. Shahid et al., "Iron, copper and silver nanoparticles: green synthesis using green and black tea leaves extracts and evaluation of antibacterial, antifungal and aflatoxin $\mathrm{B}_{1}$ adsorption activity," $L W T$, vol. 90, no. 2017, pp. 98-107, 2018.

[12] L. Muthulakshmi, N. Rajini, H. Nellaiah, T. Kathiresan, M. Jawaid, and A. V. Rajulu, "Preparation and properties of cellulose nanocomposite films with in situ generated copper nanoparticles using Terminalia catappa leaf extract," International Journal of Biological Macromolecules, vol. 95, pp. 1064-1071, 2017.

[13] S. Ahmed, M. Saifullah, B. Ahmad, L. Swami, and S. Ikram, "Green synthesis of silver nanoparticles using Azadirachta indica aqueous leaf extract," Journal of Radiation Research and Applied Science, vol. 9, no. 1, pp. 1-7, 2019.

[14] H. C. A. Murthy, B. Abebe, and T. D. Zeleke, "Material science research India a review on green synthesis and applications of $\mathrm{Cu}$ and $\mathrm{CuO}$ nanoparticles," Material Science Research India, vol. 15 , no. $3,2018$.

[15] T. Wolde, B. Bizuayehu, T. Hailemariam, and K. Tiruha, "Phytochemical analysis and antimicrobial activity of Hagenia abyssinica," Indian Journal of Pharmacy and Pharmacology, vol. 3 , no. 3, p. 127, 2016

[16] H. Tsade, B. Abebe, and H. C. A. Murthy, "Nano sized Fe-Al oxide mixed with natural maize cob sorbent for lead remediation," Materials Research Express, vol. 6, no. 8, article 85043, 2019.

[17] S. Manandhar, S. Luitel, and R. K. Dahal, "In vitro antimicrobial activity of some medicinal plants against human pathogenic bacteria," Journal of Tropical Medicine, vol. 2019, Article ID 1895340, 5 pages, 2019.

[18] M. Balouiri, M. Sadiki, and S. K. Ibnsouda, "Methods for in vitro evaluating antimicrobial activity: a review," Journal of Pharmaceutical Analysis, vol. 6, no. 2, pp. 71-79, 2016.

[19] M. Khatami, H. Heli, P. Mohammadzadeh Jahani, H. Azizi, and M. A. Lima Nobre, "Copper/copper oxide nanoparticles synthesis using Stachys lavandulifolia and its antibacterial activity," IET Nanobiotechnology, vol. 11, no. 6, pp. 709-713, 2017.

[20] N. Nazar, I. Bibi, S. Kamal et al., "Cu nanoparticles synthesis using biological molecule of $P$. granatum seeds extract as reducing and capping agent: growth mechanism and photocatalytic activity," International Journal of Biological Macromolecules, vol. 106, pp. 1203-1210, 2018.

[21] H. Le Tu, "Biosynthesis, characterization and photocatalytic activity of copper/copper oxide nanoparticles produced using aqueous extract of lemongrass leaf," Composite Materials, vol. 3, no. 1, pp. 30-35, 2019.

[22] S. Jain and M. S. Mehata, "Medicinal plant leaf extract and pure flavonoid mediated green synthesis of silver nanoparticles and their enhanced antibacterial property," Scientific Reports, vol. 7, no. 1, article 15867, 2017.

[23] K. M. Rajesh, B. Ajitha, Y. Ashok Kumar Reddy, Y. Suneetha, and P. Sreedhara Reddy, "Assisted green synthesis of copper nanoparticles using Syzygium aromaticum bud extract: Physical, optical and antimicrobial properties," Optik, vol. 154, pp. 593-600, 2018.

[24] G. Sharmila, R. Sakthi Pradeep, K. Sandiya et al., "Biogenic synthesis of $\mathrm{CuO}$ nanoparticles using Bauhinia tomentosa leaves extract: characterization and its antibacterial application," Journal of Molecular Structure, vol. 1165, pp. 288-292, 2018.

[25] R. Zia, M. Riaz, N. Farooq, A. Qamar, and S. Anjum, “Antibacterial activity of $\mathrm{Ag}$ and $\mathrm{Cu}$ nanoparticles synthesized by chemical reduction method: a comparative analysis," Materials Research Express, vol. 5, no. 7, 2018.

[26] A. Arya, K. Gupta, T. S. Chundawat, and D. Vaya, "Biogenic synthesis of copper and silver nanoparticles using green alga Botryococcus braunii and its antimicrobial activity," Bioinorganic Chemistry and Applications, vol. 2018, Article ID 7879403, 9 pages, 2018.

[27] K. Cheirmadurai, S. Biswas, R. Murali, and P. Thanikaivelan, "Green synthesis of copper nanoparticles and conducting nanobiocomposites using plant and animal sources," RSC Advances, vol. 4, no. 37, pp. 19507-19511, 2014. 
[28] M. I. Din, F. Arshad, Z. Hussain, and M. Mukhtar, "Green adeptness in the synthesis and stabilization of copper nanoparticles: catalytic, antibacterial, cytotoxicity, and antioxidant activities," Nanoscale Research Letters, vol. 12, no. 1, 2017.

[29] W. Huang, M. Yan, H. Duan, Y. Bi, X. Cheng, and H. Yu, "Synergistic antifungal activity of green synthesized silver nanoparticles and epoxiconazole against Setosphaeria turcica," Journal of Nanomaterials, vol. 2020, Article ID 9535432, 7 pages, 2020. 